Voix et Images

voixetimages

\title{
Anne Hébert et la Bible
}

\section{Antoine Sirois}

Volume 13, numéro 3 (39), printemps 1988

Jack Kerouac et l’imaginaire québécois

URI : https://id.erudit.org/iderudit/200734ar

DOI : https://doi.org/10.7202/200734ar

Aller au sommaire du numéro

\section{Éditeur(s)}

Université du Québec à Montréal

\section{ISSN}

0318-9201 (imprimé)

1705-933X (numérique)

Découvrir la revue

\section{Citer cet article}

Sirois, A. (1988). Anne Hébert et la Bible. Voix et Images, 13(3), 459-472.

https://doi.org/10.7202/200734ar d'utilisation que vous pouvez consulter en ligne.

https://apropos.erudit.org/fr/usagers/politique-dutilisation/ 


\section{Anne Hébert et la Bible}

\section{par Antoine Sirois, Université de Sherbrooke}

Anne Hébert affirme sans détour sa prédilection pour la Bible.

Interviewée sur les influences qu'elle a subies, elle déclarait: [...] la Bible est un livre extraordinaire [...]. C'est peut-être l'ouvre qui m'a marquée le plus ${ }^{1}$.

De toutes les sources auxquelles se réfere Anne Hébert dans ses récits, la Bible paraît être, en effet, la principale. Les Saintes Écritures imprègnent non seulement la civilisation chrétienne dans laquelle l'auteure a grandi, mais véhiculent, par l'Ancien Testament en particulier, des mythes orientaux millénaires à travers lesquels les écrivains sacrés ont transmis leur message, comme ceux de la création, du paradis terrestre, de la chute, de la punition, mythes auxquels l'auteure se montre très sensible.

Notre étude s'attachera aux rapports entre la Bible et les récits ${ }^{2} \mathrm{~d}^{\mathbf{A}}$ Anne Hébert. Nous verrons la fréquence, la source et la forme des références aux livres saints, pour démontrer ensuite comment le livre de la Genèse affecte la structuration des Fous de Bassan en particulier. L'analyse de la nature des références, de leur récurrence, de leur association nous révélera enfin une conception de la destinée humaine chez l'auteure, profondément marquée par les Écritures.

\section{Les références}

Anne Hébert se réfère à l'Ancien et au Nouveau Testament, plus particulièrement à certains livres saints, et selon des fréquences diverses.

Elle recourt prioritairement dans l'Ancien Testament au livre de la Genèse, ensuite quelques fois à l'Exode et aux Psaumes et enfin aux prophètes Isaie, Malachie, Daniel et au livre de Job. Les évangiles de Mathieu et de Jean sont ses sources préférées dans le Nouveau Testament, suivis de ceux de Marc et de Luc,

1 André Vanasse, «L'écriture et l'ambivalence, entrevue avec Anne Hébert», Volx \& images, vol. VII, $n^{\circ} 3$, printemps 1982, p. 446.

2 Les références aux récits sont tirées des éditions suivantes: le Torrent, Montréal, HMH, coll. «l'Arbre», 1963; les Chambres de bois, Paris, Seuil, 1958; Kamouraska, Paris, Seuil, 1970; les Enfants du sabbat, Paris, Seuil, 1975; Héloĩse, Paris, Seuil, 1980; les Fous de Bassan, Paris, Seuil, 1982. Nos citations bibliques sont tirées de la Blble de Jérusalem, Paris, les Éditions du Cerf, 1974. 
de l'Apocalypse de Jean, des épîtres de Paul, en particulier de la première aux Corinthiens. La fréquence des références, dans chacun des récits de la romancière, dépend de leur contenu, de leur thématique, mais on remarque un accroissement de la fréquence des citations comme telles du premier récit au dernier. Si l'on n'en rencontre que quelques-unes dans le Torrent et les Chambres de bois, en revanche, on en relève un nombre plus élevé dans Kamouraska, une abondance dans les Enfants du sabbat, dont l'histoire se déroule en grande partie dans un couvent de religieuses, et dans les Fous de Bassan. Dans ce dernier roman en particulier, on remarque une cinquantaine de citations dans la bouche surtout du pasteur d'une petite communauté protestante isolée, sans compter une cinquantaine d'autres renvois aux livres saints. Le texte fondateur pour l'ensemble de l'œuvre est celui de la Genèse (ch. I-IV surtout) auquel se réfêre du reste le Torrent dès 1950, par des énoncés de grands thèmes qui s'en inspirent comme le péché originel, la justice, le châtiment, plutôt que par des citations explicites. Les renvois aux autres livres saints auront souvent un rapport à la chute de David, dans les Psaumes, ou au jugement dernier, dans les Évangiles ou au combat du bien et du mal, dans l'Apocalypse.

Les références à la Bible revêtent des formes très variées. Elles consistent d'abord en de simples allusions ou en des renvois plus élaborés à des personnages, thèmes ou motifs.

Anne Hébert donne des rôles multiples, dans sa narration, aux personnages référents. Ils peuvent servir de modèle de comportement: François est invite par sa mère Claudine à s'immoler comme le Christ (T20), Joseph baptise sa sœur Julie dans la rivière comme l'a fait Jean-Baptiste pour le Seigneur (ES154). Ces modeles, d'ailleurs, peuvent être archétypaux, comme Ėve, le Serpent, et leur comportement ou leur situation se reproduire de façon analogue dans le récit contemporain. Les personnages ont parfois l'impression de les imiter dans leurs actions comme François qui, comme Jacob, croit livrer son propre combat contre l'Ange (T36). Le référent sert aussi de terme de comparaison: Hitler est comparé à l'Antéchrist (ES153), les frères de sœur Catherine se mourant de désespoir seront considérés comme le bon et le mauvais larron (K170). L'imitation peut être poussée assez loin èn ce sens que certains personnages jouent un rôle qui non seulement se modèle sur l'original, mais aussi le prolonge en quelque sorte dans son action. Stevens Brown est, pour sa cousine Olivia, l'arbre personnalisé de la science du bien et du mal duquel elle peut tout apprendre (FB216). Les sorciers Adélard et Julie, complices du diable, poursuivent la lutte de Lucifer contre Dieu (ES107, 141).

Les renvois à des personnages, lieux, situations bibliques peuvent être aussi parodiques. On en trouve des utilisations fort manifestes dans les Enfants du sabbat avec la célébration de la messe noire. Les paroles exactes de la consécration sont prononcées mais inversées dans leur ordre. L'immolation est celle d'un petit cochon (ES43). Julie sera promenée, revêtue, comme le Christ aux mains des soldats, d'une couronne et d'un manteau pourpre (ES69). La malfaisante Elisabeth, dans Kamouraska, veut se débarrasser de son époux et 
déclare: Il faut qu'Antoine meure et que je sois sauvée de la mort... Il faut que George soit sauvé. Par la mort d'Antoine. Célébrons ce sacrifice (K167).

Les références consistent, en second lieu, en des citations. Nous pourrions rappeler ici la notion de transtextualité exposée par Genette dans Introduction a l'architexte ${ }^{3}$. Elle englobe pour lui l'intertextualité au sens strict: la citation, plus ou moins littérale, intégrale ou non, et une autre forme de relations appelée paratextualité et qui sont imitation et transformation comme le pastiche et la parodie.

La notion de paratextualité pourrait s'appliquer à la forme de référence que nous venons d'exposer, surtout celle qui est renvoi à des personnages et des situations bibliques, en particulier à des actions prolongées comme celle de la messe noire (ES43, 44). L'immolation du Christ à la Cène et sur la croix, telle que racontée dans le Nouveau Testament, est transposée, sous un mode parodique, à l'intérieur du récit. Il s'agit là d'un segment parodique qui, tout en contenant même des citations au texte (les paroles de la consécration), constitue une forme de renvoi qui dépasse la simple citation.

Dans ce que nous abordons maintenant, nous traitons d'intertextualité, de citations dans un sens plus strict. Elles revêtent, dans l'œuvre d'Hébert, des formes fort variées. Certaines sont exactes, mot à mot, d'autres, modifiées, transformées, tronquées, parfois même occultées. Les citations au texte se présentent habituellement entre guillemets ou en italique. Elles peuvent garder leur sens religieux, correspondant à la situation contextuelle.

Par sens religieux, nous entendons le sens de l'Écriture elle-même, qu'un lecteur interprète pour en recueillir le message originel. Mais ce sens est du reste multiple: littéral, spirituel, accomodatice 4 . Les extraits des Psaumes, chantés par les religieuses dans les Enfants du sabbat $(104,106)$, expriment leur prière et les passages auxquels recourt le pasteur Nicolas Jones dans les Fous de Bassan $(22,24,27)$ semblent influencer son comportement moral et lui font dire: Les épîtres de Paul, le livre de Jean, la contre ma main, comme si on pouvait sentir le souffle des apôtres rien qu'en posant les mains sur la couverture de cuir noir (22). La citation pourrait garder un sens religieux, mais avoir une application dite accomodatice, en ce sens que le narrateur adapte le passage sacré afin d'illustrer une situation donnée. Le narrateur des Fous de Bassan, pour décrire la situation idyllique vécue d'abord par une petite colonie loyaliste,

3 Gérard Genette, Introduction à l'architexte, Paris, Seuil, 1979, p. 87-88.

4 Il y a traditionnellement quatre sens à l'Ériture: le sens premier, littéral ou historique, le sens spirituel qui se répartit lui-même en trois: sens allégorique, sens moral (le faire), sens anagogique. Ce demier est un sens ultime qui résulte du symbolisme par lequel ces choses figurent ce qui a lieu dans la gloire éternelle. Voir: Antoine Compagnon, la Seconde main ou le Travall de la citation, Paris, Seuil, 1979; Paul Toinet, Pour une théologle de l'exégèse, Paris, Fac-édition, 1983. Les Ecritures sont soumises aussi aux mêmes approches critiques que les textes littéraires. 
rappelle une parole s'appliquant aux temps premiers et cite alors: Et l'esprit de Dieu planait sur les eaux (K14-Gn1.2). Ces citations au texte fonctionnent un peu comme des épigraphes qui viennent donner un sens à un passage. Elles s'intègrent de façon pertinente car elles font partie souvent de la réflexion du pasteur. Mais une citation entre guillemets peut aussi s'accomoder à une action qui n'a rien de religieux. Georges Nelson, qui veut se gagner la femme de son rival démuni, déclare: À celui qui n'a rien, il sera encore enlevé quelque chose (K129-Mt25.29).

Les citations au texte sont encore employées d'une façon totalement parodique, comme nous l'avons vu antérieurement pour le Ceci est mon corps appliqué à la consécration d'un cochon (ES43). Des citations en italique pourront comporter un léger changement qui, en quelque sorte, pervertit le texte religieux. Le «il» se tansforme en «elle» et ce qui était dit du Serviteur de Yahvé dans Isaïe se rapporte à une religieuse accablé: Elle n'a plus d'apparence. C'est qu'elle porte nos péchés... (K82).

Habituellement les citations altérées, souvent parodiques, sont en romain et s'intègrent au texte courant. La sorcière Julie reprend le texte de Jean-Baptiste parlant du Christ en proférant: Il faut que je croisse et que ma mère diminue (ES106). Julie évoque son frère Joseph dont elle est amoureuse en disant: Le plus beau parmi les enfants des hommes (ES24), et rappelle ainsi le verset 5 du psaume 45 appliqué au Messie: Tu est beau au-dessus des enfants des hommes. Nous avons encore le cas d'une citation au texte, en italique, religieuse dans son sens premier, qui se transforme, en romain, dans un sens profane. Le péché est tapi à la porte, son élan est vers toi... (FB17-Gn4.7) devient, quelques pages plus loin: [...] une petite bête lustrée, d̀ l'affût dans l'herbe (FB73), pour traduire l'appétit de la petite cousine pour Stevens. La même image profane apparaissait déjà dans Kamouraska quand Elisabeth se remémorait avec angoisse la maison de son enfance et la vie tumultueuse appréhendée: Une bête sauvage qu'on a enfermée et qui guette dans l'ombre pour vous sauter dessus (51). Michel était aussi cette bête aux aguets qui épiait Catherine (CB58).

On trouve encore des citations agglutinées composées de deux passages tronqués et réunis dans une seule phrase. Encore un peu de temps et tout sera consommé (ES144) provient de: Encore un peu de temps et vous ne me verrez plus (Jn16.17) et de Tout est consommé (Jn19.30). Des mots nouveaux peuvent être insérés dans une phrase biblique tronquée. Je suis l'amour et la vie émane de Je suis la voie, la vérité, la vie (Jn14.6). La parole du Christ s'adressant aux riches se prête à un jeu de mots: Le royaume du ciel est pour le chat qui réussit à passer par le trou de l'aiguille (ES140). On pourra ajouter la citation suivante comme modèle de parodie et d'inversion: Bénis sommes-nous par qui le scandale arrive (K131) pour [...] malheur à l'homme par qui le scandale arrive (Mt18.7). Enfin la référence peut exister, mais doit se laisser deviner. Se cache derrière: L'univers saura que le mal m'a choisi dès le premier souffle de mon existence (T63) l'original suivant: Vois: mauvais je suis né, pécheur ma mère m'a conçu (Ps51.7). On comprend que d'une part Anne Hébert a bien assimilé sa Bible et 
que, d'autre part, le repérage, par le lecteur, de plusieurs citations bibliques exige une grande familiarité avec les livres saints.

Mais que la citation biblique soit littérale ou transformée, religieuse ou profane, elle est toujours récrite par l'auteure. Celle-ci reprend, choisit les passages qui l'ont sollicitée, se les approprie, les plie à son propos. Ces extraits, puisés dans les textes sacrés, se parent tout de même de leur résonance, de leur sagesse, de leur autorité.

\section{Le *pattern» des Fous de Basssan}

La romancière ne fait pas que citer, intégrer savamment des textes sacrés dans sa narration, elle ira même jusqu'à structurer un des ses romans, les Fous de Bassan, à partir d'un des livres, celui de la Genèse. La romancière ellemême, dans une interview, justifiait la vraisemblance de ce roman et ses abondantes références à la Bible en déclarant que la culture que possèdent ses personnages leur vient surtout de la fréquentation quotidienne de la Bible; c'est la Bible qui leur fournit un langage très imagé et une sorte de grandeur qui les dépasse 5 .

Résumons d'abord l'intrigue pour la bien comprendre.

Stevens Brown, vingt ans, revient, après plusieurs années de voyages à travers l'Amérique, à son village natal, Griffin Creek, concession sur les bords du Saint-Laurent faite en 1782 à un groupe de loyalistes américains. La communauté y mène une vie relativement paisible sous la houlette du pasteur Nicolas Jones. Mais voilà que dans la nuit du 31 août 1936 deux adolescentes, Nora et Olivia Atkins, disparaissent. Que leur est-il arrivé? La mer finira par ramener des indices de leur mort violente et l'enquête policière révélera la culpabilité de Stevens. Le village se désintègre, les habitants se dispersent. Le pasteur vieillira seul au milieu des survivants et Stevens, après avoir échappé à la condamnation et s'être enrôlé, se retrouvera après la guerre, détraqué, dans un hôpital pour vétérans.

Cette histoire nous est présentée de façon polyphonique, en six récits, par différents acteurs du drame qui nous apportent chacun leur point de vue, sous différentes formes. Même le fantôme de Nora viendra témoigner.

À partir de ces six récits nous pouvons reconstituer chronologiquement le drame, en dégager les principales étapes, et relever les références bibliques qui s'y rapportent.

L'histoire remonte au commencement (14), selon les termes même de la Genèse, et décrit un espace et un temps primordiaux de façon analogue au récit biblique: Au commencement, il n'y eut que cette terre de taïga... toutes les bêtes

5 Brigitte Morissette, «Lointaine et proche, Anne Hébert», Chatelaine, février 1983, p. 50 . 
à fourrures et d plumes... les oiseaux de mer et les poissons dans l'eau s'y multipliaient à l'infini. Et l'esprit de Dieu planait au-dessus des eaux (14-Gn1.2).

Cette terre promise à laquelle ont accédé les loyalistes après leur pérégrination, ce jardin d'abondance, abrite deux pommiers, comme au chapitre deux de la Genèse, mais qui donnent ici des pommes acides que les cousines jumelles ont croquées avant leur mort violente $(134,157)$. On y trouve également deux personnages qui sont assimilés à l'arbre de la connaissance du bien et du mal (Gn2.8) $(119,216)$, le pasteur Nicolas Jones et le jeune Stevens Brown, qui convoitent chacun les deux adolescentes désirables, Nora et Olivia. Stevens, ce diable d'homme (106), ressemble aussi au tentateur $(103,104)$, se voit comparer à un serpent $(77,214)$ et se retrouvera perdu dans l'enfer de Griffin Creek (243). Quant au pasteur, aiguillonné par la concupiscence, évoquée par l'apôtre Paul dans sa première épître aux Corinthiens: Mieux vaut se marier que de brûler (1Co7.9), il nous ramène aussi à l'époque du nouvel Adam, le Christ, dont luimême est l'image (40). C'est à l'origine du Nouveau Testament que nous sommes ici conviés, au Verbe s'incarnant dans le monde, dans les premiers versets de l'évangile de Jean. Le lien est ici parodique, car Nicolas, prédicateur infidèle de la Parole, devient le Verbe qui s'est fait chair, mais dans un sens péjoratif, et il habite désormais parmi le commun des hommes (18, 19-Jn1.14).

Dans le jardin de Griffin Creek déambulent les deux femmes de soleil, cihaut évoquées, Olivia et Nora. Cette dernière se compare elle-même à une Ève nouvelle (118) qui se voit faite du limon de la terre, comme Adam, et non sortie d'entre les côtes sèches d'Adam, première comme Adam, conformément au premier récit de la création (116-Gn1.27). À l'exemple de la première Ėve, elle est avide de connaître (116) la révélation de l'arbre défendu, (Gn3.6). Olivia éprouve la même tentation. Elle dit de Stevens:

Il est comme l'arbre planté au milieu du paradis terrestre. La science du bien et du mal n'a pas de secret pour lui. Si seulement je voulais bien j'apprendrais tout de lui, d'un seul coup, la vie, la mort, tout. Je ne serais plus jamais une innocente simplette... l'amour seul pourrait faire que je devienne femme à part entière et communique avec mes mères et grands-mères... du mystère qui me ravage, corps et âme. (216)

Tout est en place pour «la chute». Le fruit à croquer, dans ce drame contemporain, c'est celui du sexe et l'innocence de la nouvelle Ève est ici relative. Elle est pleine de désirs, mais n'a pas encore connu l'expérience. Le jeu de la tentation, pour celle qui est à la fois désirante et désirée (215), se déroule dans le regard. Elle a levé les yeux vers l'homme et elle a été prise dans le regard de Stevens comme dans un filet (216). La rencontre des deux adolescentes avec le garçon sur la grève, le soir du trente et un août, débouche sur le sexe, la violence et la mort. Stevens tue d'abord Nora qui, frustrée par l'apparente indifférence de celui-ci, le nargue sur sa virilité et ensuite Olivia, pour qu'elle ne le dénonce pas, mais après l'avoir pénétrée comme un bourdon au cœur d'une pivoine (246). 
Tout est parallele, mais aussi analogie, entre les actions de Griffin Creek et celles du jardin d'Éden. Le mal, tel que signalé plus haut, ne fait pas son apparition pour une première fois dans cette communauté. Comme le dit le pasteur:

[je] cherche la faute originelle de Griffin Creek. Non, ce n'est pas Stevens qui a manqué le premier, quoiqu'il soit le pire de nous tous, le dépositaire de toute la malfaisance secrète de Griffin Creek, amassée au coeur des hommes et des femmes depuis deux siècles (27).

Stevens a agi comme détonateur en franchissant le seuil de l'intolérable (52).

À la faute succédera la punition; le rapport entre la Bible et le roman se poursuit. Maintenant le jardin de Maureen est envahi par les mauvaises herbes, celui du pasteur sent l'ail et le poireau, les épilobes foisonnent dans les champs en friche (199). L'Éden a perdu de son attrait, la période d'abondance est terminée, les constructions se délabrent et les habitants quittent (52). Il a suffi d'un seul été, comme le dit Nicolas, pour que se disperse le peuple élu de Griffin Creek (13).

Le destin des deux adolescentes trop curieuses et victimes des forces du mal est scellé. Le sort qui échoit à Stevens en particulier ressemble à celui de Caïn qui s'était fait dire par Yahvé après son crime: Sois maudit et chassé du sol fertile qui a ouvert la bouche pour recevoir de ta main le sang de ton frère (Gn4.11). Lui, meurtrier de ses cousines, affirme dans sa dernière lettre: Hommes et femmes de Griffin Creek... se lèvent pour me maudire. Me chassent de Griffin Creek (240).

Le pasteur, vieux et décrépit, songera avec nostalgie à autrefois lorsque le monde était innocent (54), et pleurera sur sa faute, comme David, au psaume cinquante et un, en attendant le Jugement. Dieu seul pourra me laver de l'ombre de ma faute et tout Griffin Creek avec moi que je traîne dans l'ombre de ma faute (47-48). L'ensemble de la collectivité est marquée par «la faute».

Quand le Jugement final viendra, et là la romancière noús reporte à l'Apocalypse de Jean (8.2-6), l'ange d'apocalypse qui soufflera dans la trompette sera, dans la vision du pasteur, Perceval, l'idiot, l'innocent du village, avec sa tête de chérubin (51).

En déroulant la trame du récit nous avons pu faire état du parallèle qui courait entre la Bible et ce récit, entre la Genèse d'abord et les événements contemporains. Pour important que soient ce parallélisme des actions, des situations, des personnages, l'emprunt des images et des symboles, l'intégration du langage biblique dans le langage quotidien des habitants du village que nous avons signalés jusqu'ici, ils n'épuisent pas les références à la Bible.

Ce que nous voulons surtout souligner, c'est le «pattern» qu'offre au récit actuel la première partie de la Genèse portant sur la création, la tentation, la 
chute, le jugement et la punition. Selon les catégories de John White dans Mythology in the Modern Novel 6 qui distingue roman mythique, qui est re-narration d'un mythe, et roman mythologique, nous croyons avoir affaire, dans les Fous de Bassan, à un roman qui serait de type mythologique. Nous trouvons dans le roman non seulement des allusions nombreuses à la Bible, mais celle-ci offre au récit actuel une préfiguration, un modèle de déroulement clairement identifié, un «pattern» de lecture. L'analogie entre les événements primordiaux et les événements modernes est prolongée et relativement complète, à partir des commencements innocents jusqu'à la punition. Le récit ancien fournit un schéma fondamental aux principales actions du récit contemporain, sans que le tracé soit nécessairement suivi dans le développement narratif à cause des procédés que la romancière utilise et qui ne permettent pas toujours un ordre chronologique. Le «pattern» ancien apporte aussi, avec toutes les variantes, les adaptations, les transformations, les transpositions que supposent le récit actuel et la liberté du créateur, une forme d'éclairage à la vision du monde de la romancière.

\section{La vision du monde}

Nous aimerions démontrer dans cette troisième partie qu'Anne Hébert emprunte à la Bible non seulement des passages sous des formes variées et un «pattern» pour les Fous de Bassan en particulier, mais une conception personnelle de la destinée humaine qu'elle traduit dans le sort réservé à ses personnages. Déjà, nous avons pu percevoir, dans les textes évoqués et l'analyse plus systématique des Fous de Bassan, que certains thèmes bibliques parcouraient les récits et sous-tendaient l'œuvre dans son ensemble.

Les thèmes majeurs nous sont présentés directement et sans fard par la bouche de la grande Claudine, hérö̈ne du premier récit majeur, le Torrent, quand elle rappelle à l'autre protagoniste principal, son fils, et en prononçant chaque syllabe, les grandes vérités: «Châtiment», «justice de Dieu», «damnation», «enfer», «discipline», «péché originel», et quand elle lui répète, comme leitmotiv: Il faut se dompter jusqu'aux os. On n'a pas idée de la force mauvaise qui est en nous! $(10,11)$.

Justice de Dieu, Dieu de justice. Cette conception de Dieu se représentera maintes fois et contribuera à la désespérance de François. C'est à ce Dieu intraitable qu'il faudra rendre compte de ses actes au jour de sa justice (23), au Jugement dernier (55). Il voudrait croire en sa droiture terrible, en sa parfaite grandeur (60), mais le Dieu qu'on lui a présenté ne correspond pas à l'image qu'il aurait aimé s'en faire. Très tôt je fus détourné de la saveur possible de Dieu (56), dit-il, et il ne connaîtra de lui que des signes vides. Même ses prières sont conçues comme faisant rempart contre l'ombre possible de la face nue de Dieu (24). De semblables perceptions se retrouvent dans d'autres romans comme

6 John J. White, Mythology in the Modern Novel, Princeton University Press, 1971. 
Kamouraska où Elisabeth demande à George, qui avait déjà rêvé d'absolu avant d'être envahi par des pensées criminelles: Quel Dieu noir a reçu tes voux (205), ou comme les Enfants du sabbat où scur Gemma est en attente du feu de Dieu... l'anéantissant jusqu'd la dernière parcelle de son être (144). Le pasteur du dernier roman, les Fous de Bassan, se prépare à sa rencontre face à face, au Jugement, et sa faute sera sur sa face comme une ombre.

Dieu de justice. Dieu d'apocalypse qui hante les personnages. Ceux-ci auront à vivre dans un monde manichéen où s'opposent à Dieu les forces du mal, incarnées en Satan. Alors que François, du Torrent, voudrait croire à Dieu et à sa droiture terrible, c'est une nouvelle Ėve, Amica, qui l'en détourne. Le diable est donc bien puissant! Et je suis son complice (60), se dit-il. Des personnages diaboliques réapparaissent dans chaque roman, identifiés comme tel, sauf dans les Chambres de bois où la qualification est implicite. Il s'agit de Lia, qui est devenue de la boue pour son frère. La faute est entrée chez nous avec elle (60), précise Michel. On reconnaîtra dans cette citation une référence à l'êpitre de saint Paul aux Romains, référence qui nous semble fournir une clef à l'œuvre de la romancière. Le texte se lit comme suit: Ainsi donc, comme par un seul homme le péché (avec un article en grec et donc personnifié) est entré dans le monde, et par le péché la mort... (5.12). Tout se déroulera dans l'œuvre comme si ce texte n'avait pas sa contrepartie d'espérance que pose peu après l'Apôtre au verset 18: Ainsi donc comme par la faute d'un seul la condamnation est venue sur tous les hommes, ainsi par la justice d'un seul (le Christ) vient à tous les hommes la justification qui donne la vie.

Le tentateur qui a fait chuter le premier homme poursuit inlassablement son œuvre et se manifeste ouvertement dans les sorciers et sorcières qui ont conclu des pactes avec lui, et de façon éclatante dans Adélard, Philomène et leur fille Julie des Enfants du Sabbat. Celle-ci voit le diable en son père qui célèbre la messe noire et la viole. Sa mère lui murmure à l'oreille: C'est l'Arbre de Science, l'Arbre de Vie, le serpent qui a vaincu Dieu qui se trouve à présent planté dans ton corps... Le diable, ton père, t'a engendrée une seconde fois (en lui passant les pouvoirs) (69). Quand Julie sortira du couvent, elle affirmera: Je leur ai donné le démon à communier [à l'aumônier et à la supérieure]. Le mal est en eux maintenant... Je n'ai plus rien à faire dans cette maison. Mission accomplie. Mon maître sera content (187). Satan qui, selon le texte énoncé antérieurement, semble conduire victorieusement sa lutte contre Dieu, n'en est pas moins un être châtié éternellement, comme le découvre la supérieure dans le grenier du couvent d'où provient une plainte continue. Elle voit

le désespoir de tout près... «La peine du dam», pense mère MarieClotilde. Elle regarde, ne pouvant s'empêcher de regarder, quoique cela soit plus insoutenable qu'aucune vision du Christ crucifié. Un corps, moitié enfant, moitié animal, torturé durant l'éternité. Une tête de petit bouc d qui on a limé les cornes. Mère Marie-Clotilde sait qu'elle se trouve en présence du démon... (181). 
Aurélie Caron, la bonne du personnage central, Elisabeth, sera la sorcière dans Kamouraska et le docteur Nelson, l'amant à l'œil noir d'Elisabeth, la personnification du diable, le roi des démons (194), dit la sorcière7. Il assassinera dans le sang le mari légitime. Hélö̈se, du roman du même nom, est une vampire manipulée par Bottereau, ce vieux démon (54). Tous deux tueront Christine et Bernard. Ce dernier, poursuivant la vampire dans le métro de Paris (associé aux enfers), fait un arrêt momentané à la station Michel-Ange-Auteuil, dont le nom s'étale sur la muraille, blanc sur bleu, précis et rassurant (120). C'est un rappel de la lutte entre le bien et le mal, l'ange Michel étant celui qui, dans l'Apocalypse, mène la lutte contre le dragon (Satan) qui menace la femme enceinte (la Vierge rappelee par le blanc sur le bleu), qui allait enfanter. Le texte de Jean (Ap12.4-12) se lit comme suit:

En arrêt devant la Femme en travail, le Dragon s'apprête à dévorer son enfant aussitôt né. Or la femme mit au monde un enfant mâle, celui qui doit mener toutes les nations avec un sceptre de fer... Alors il y eut une bataille dans le ciel: Michel et ses anges combattirent le Dragon... On le jeta donc, l'énorme Dragon, l'antique Serpent, le Diable ou le Satan, comme on l'appelle, le séducteur du monde entier, on le jeta sur la terre. Malheur à vous la terre et la mer, car le Diable est descendu chez vous, frémissant de colère...

La romancière nous avait renvoyés deux fois à ce passage dans les Enfants du Sabbat, quand la supérieure eut la conviction que sœur Julie était possédée (141-Ap12.12), et quand la sorcière voulut faire la célébration de l'inceste avec son fils, disant: Telle est la loi, afin que naisse, et du fils et de la mère, le plus noir génie jamais promis au monde. La possession de la terre sera pour ce fils unique. Il fera paftre toutes les nations avec une trique de fer (107Ap12.5). Cette demière citation biblique, reprise par Philomène, correspond exactement à une section du passage rapporté plus haut, sauf que le mot «sceptre» est remplacé par le mot «trique». Au Christ est opposé un antéchrist. La lutte se poursuit inlassablement et les humains en sont les victimes.

Dans le roman le plus récent qui, comme nous l'avons vu, est une réactualisation du drame de la Genèse, le démon a toujours sa place, manifesté surtout par Stevens, diable et serpent, qui entraîne ses cousines, puis les viole et les tue.

Mais l'homme se montre facilement complice de Satan; il est habité par le mal, dont le diable est la cause et la figure par excellence. Nous nous souvenons que les mots «péché originel» figuraient dans les mots clefs dont la mère accablait le fils dans le Torrent. Claudine avait repris ces mots sous une autre

7 Il est intéressant de souligner que certains traits attribués à Nelson se retrouvent dans le personnage du diable présenté dans un vieux conte canadien «Rose Latulipe»: yeux noirs, revêtu d'un manteau de chat sauvage, conduisant un cheval noir. Voir le Chercheur de Trésor de Philippe-Aubert de Gaspé. 
forme: On n'a pas idée de la force mauvaise qui est en nous (11). Elle intimait à François, plus loin: Tu combattras l'instinct mauvais jusqu'à la perfection (19). La même idée refait surface lorsque le jeune homme dit: Tout homme porte en soi un crime inconnu qui suinte et qu'il expie (55). Il y avait allusion à la première faute dans les Chambres de bois, lorsque Michel énonçait que la faute était entrée chez eux avec Lia, ce qui rappelait: [...] par un seul homme le péché est entré dans le monde... (Rm5.12), et renvoi évident dans Kamouraska, alors que l'on récitait les prières des agonisants tirés en partie du psaume 51 de David: Vois, dans le mal je suis né. Pécheur ma mère m'a conçu (5-8). Ce dernier passage, avec celui que nous venons de citer de l'Épître aux Romains, constituent une partie importante des textes fondamentaux auxquels se réfere l'exégèse touchant le péché originel, dont la nature exacte est du reste controversée. Mais il semble bien impliquer chez Anne Hébert que nous sommes tous marqués par le péché et le mal depuis l'origine. Le narrateur s'y réfêre encore clairement quand, dans les Enfants du sabbat, il dit de sœur Gemma, alors qu'elle est sacristine: Rien ne semblait peser sur elle. Aucun péril, misère ou faute, ni même le péché originel (46), ou qu'il fait dire à l'aumônier et à la supérieure du couvent qui veulent faire disparaître l'enfant mis au monde par sœur Julie: Nous paierons notre dette a Dieu, ou au diable, peut-être même l'avonsnous payée d'avance? Tant de prières et de sacrifices, depuis le commencement des temps, et l'obscure faute originelle à racheter... (185). Le pasteur dans les Fous de Bassan ne manque pas de parler de la faute originelle de Griffin Creek qui consiste, dans ce roman, dans la malfaisance amassée au caur des hommes et des femmes depuis deux siècles (27). L'emploi de «faute originelle», même si celle-ci, dans le cas présent, ne remonte pas aux origines de l'humanité, permet au moins de percevoir l'id́́e que se fait la romancière de la tendance héréditaire et cumulative du mal qui marque les humains.

Non seulement les humains sont marqués par un mal héréditaire au dedans d'eux-mêmes, tapi comme une bête voulant les dévorer, mais ils font sans cesse face à des tentateurs, leurs congénères: Amica pour François, Stevens pour ses cousines, Elisabeth pour George, Michel pour Catherine, Héloïse pour Bernard, Julie pour sa communauté.

La propension au mal se manifeste souvent par le désir qui est l'appétit de l'autre sexe et qui est fatal. François le traduit ainsi: Ce désir de la femme m'a rejoint dans le désert... C'est impitoyable... Posséder et détruire et le corps et l'âme d'une femme... Et voir la femme tenir son rôle dans ma propre destruction (T29). L'innocente jeune fille des Chambres de bois donne asile au rêve... pleine de défi et de mystère comme celle qui flaire un prince barbare en secret (39). Elle passe très près d'y trouver la mort, soumise longtemps au désir morbide de Michel. Elisabeth, qui connaît l'homme dès quinze ans, manifeste clairement son attirance vers le premier qu'elle connaîtra, sans vraiment l'aimer: Le centre de ma vie, ce désir... Dans le brasier de l'été (K71-72). C'est encore Nora et Olivia qui, malgré toutes les mises en garde de leurs ainées, désirent devenir femme par l'amour, et pour leur plus grand malheur. Olivia parle même du mystère qui la ravage, corps et âme (FB216). Cette tentation se réalise 
souvent par le regard, motif dominant dans l'œuvre d'Anne Hébert, saisissant sa victime comme dans un filet.

La grande Claudine avait repris pour son fils la phrase de la Genèse: Son envie (le péché) le pousse vers toi, mais toi tu dois le dominer (4.7). Cette maîtrise, ce contrôle, l'homme paraît difficilement l'exercer. Le mal s'abat plutôt sur lui comme une fatalité. François semble bien exprimer, dès le Torrent, la condition humaine sans espoir:

Tout d'un coup j'ai été en face du gouffre intérieur de l'homme, je m'y suis abimé. De mon vivant je goûte au jugement dernier: cette confrontation réelle avec soi, c'est trop pour les forces humaines. Je brûle... Parfois il me vient une pensée qui pourrait être un allégement, une grâce, si je pouvais croire d̀ l'apaisement et si la grâce ne m'était pas refusée (55).

Il n'y a que la Catherine des Chambre de bois qui connaîtra une résurrection comme la fille de Jaire (152-Mc5.42). Mais les autres subissent la fatalité du mal qui les habite et l'innoncence qu'ils peuvent tenter d'acquérir, comme Elisabeth dans Kamouaska, est une innocence de surface, de façade, un masque pour les yeux d'autrui.

L'homme, coincé entre un Dieu froid qui ne l'agrée pas et un Satan maléfique qui s'acharne à l'entraîner dans sa chute, souillé lui-même par le péché, est condamné à expier. Tous les personnages principaux sont en perpétuel état d'immolation et se sacrifient les uns les autres. L'exemple vient de haut, du fils même de Dieu. Claudine dit à son fils, qu'elle veut le voir accéder à la prêtrise: Le prêtre est à la fois sacrificateur et victime comme le Christ. Il fallait qu'il s'immolât sur l'autel, sans merci, avec l'hostie (T20). C'est le sort de tous, puisque François, plus tard, précise: Tout homme porte en soi un crime inconnu et qu'il expie (T55).

Les victimes sont très variées et dans des situations fort différentes. C'est le sort des religieuses dont la vie vient mourir au couvent, de l'aumônier et de la supérieure du même couvent dont la pénitence sera sans fin, dans les Enfants du sabbat. Et la même fatalité est réservée à la sœur Catherine des Anges, dans Kamouraska, dont la tendresse est immolée dans sa source. Ce malheur peut être aussi la destinée de personnages considérés comme maléfiques ou diaboliques, tel l'assassin d'Antoine, George, qu'Elisabeth a vu comme crucifié à un arbre et qu'elle laissera tomber; tel Antoine lui-même, coureur de jupons, qui périt sous le couteau de George dans le sang. Michel fera de Catherine une victime qui devient pareille d une jeune offrande sur la table de pierre, et Héloïse et Bottereau immoleront Bernard et Christine, jeunes époux que Bottereau avait appelé mes agneaux. Dans chacun des romans, un ou des personnages sont immolés par les autres. Et les instruments du sacrifice traditionnel, comme l'autel et l'arme tranchante, reviennent avec fréquence. L'autel peut être un lit, comme pour Catherine, exposée en sa passion sur un lit de parade, et dont la bonne célèbre les 
offices autour de son lit, comme pour Satan lui-même, découvert par la supérieure, souffrant la peine du dam, couché sur un lit de fer. L'autel sera encore une table, celle où Julie sera offerte et consacrée par ses parents. Un instrument tranchant sacrifiera Antoine, Bemard et Christine, et le cochon de la messe noire.

Plusieurs des victimes font fonction de boucs émissaires. Ce rite millénaire, que l'on retrouve dans l'Ancien Testament, se manifeste d'abord dans le Torrent, quand François est invité à devenir prêtre et à s'immoler comme le Christ, ou dans Kamouraska, alors qu'Elisabeth réclame qu'Antoine meure et qu'elle soit sauvée par sa mort, mais surtout dans les Enfants du sabbat où il connaît une sorte de couronnement. Adélard, entouré de chômeurs accablés par la misère, sacrifiera parodiquement le cochon pour se rendre Satan propice, et symboliquement sa fille, et enfin sa femme, en punition pour avoir raté l'initiation de son fils. Les villageois à leur tour, pour tenter de retrouver une certaine innocence, sacrifieront la cabane, dans laquelle les sorciers pourraient se trouver, en y mettant le feu. Dans ce même roman, on appliquera à sour Gemma les paroles destinées au Serviteur souffrant: C'est qu'elle porte nos péchés. Elle est transperçée à cause de nos péchés (82). La supérieure transposera pour sœur Julie les paroles de Caiphe sur le sort de Jésus: Ne vaut-il pas mieux qu'une seule de mes filles périsse et que toutes les autres soient sauvées (141).

Contrairement à la vision biblique, Anne Hébert propose une vision tragique du sort de l'homme auquel ne s'offre aucun espoir, aucun salut véritable. Tout homme porte en soi un crime inconnu qui suinte et qu'il expie (T55). La deuxième partie de l'alternance proposée par saint Paul n'a pas été retenue. Au verset: ainsi donc, comme par un seul homme le péché est entré dans le monde, et par le péché la mort...., ne succède pas dans l'œuvre le second verset: ainsi par la justice d'un seul vient d tous les hommes la justification qui donne la vie (Rm2.12-18). Échoit à l'homme le sort de Caïn après son crime. Il portera sa punition de par le monde, poursuivi par la vindicte divine. Le péché, couché à sa porte, est toujours prêt à bondir sur lui, et Satan, chassé des cieux, est descendu sur lui avec une grande fureur. Victime perpétuelle, il s'immolera lui-même, sacrifiera les autres ou sera sacrifié par eux.

La romancière a surtout utilisé les passages bibliques terrifiants qui alimentent son vocabulaire et meublent son imaginaire d'images et de symboles qu'elle a habilement insérés dans ses récits.

Nous pouvons déduire de cet exposé qu'Anne Hébert entretient dans son œuvre une représentation vétéro-testamentaire, axée sur la chute et le Dieu-juge, qui n'est pas corrigée par le message plus optimiste du Nouveau Testament sous le signe du Dieu-sauveur.

L'univers symbolique d'Anne Hébert a fait l'objet d'une étude approfondie de Maurice Émond, dans la Femme à sa fenêtre ${ }^{8}$. Selon lui, les images de chute

8 Émond, Maurice, la Femme à sa fenêtre, Québec, Les Presses de l'Université Laval, 1984. 
sont nombreuses. Il en arrive à une conclusion qui concourt avec la nôtre. Les personnages hébertiens, écrit-il, sont marqués par la notion de faute originelle, traumatisés par la crainte du châtiment suprême. Ils sont moins préoccupés du ciel que de l'enfer qui en vient d exercer sur eux une véritable fascination (75). Ils vivent aussi, comme l'ont remarqué plusieurs analystes, dans un monde manichéen. Tout est dualité dans l'œuvre: bien et mal, lumière et ténèbres. Les références à la Bible soulignent ce combat entre deux forces, divines et sataniques, entre lesquelles les humains sont déchirés et éprouvent, malgré leur désir de vie et leur nostalgie de l'enfance et de l'innocence, une forte propension vers le mal et la mort, et un besoin continuel d'expiation.

\section{Le pourquoi}

Pour quelle raison Anne Hébert a-t-elle retenu cette optique? Comme elle le déclare dans une interview: J'ai été très marquée par la religion de mon enfance. Je la rejette d'une certaine façon, mais elle est en moi et en la rejetant, je ne peiux pas rejeter toute une culture ${ }^{9}$. Elle déplore la morale figée de cette époque des années trente. Elle semble avoir surtout gardé l'image d'un Dieu justicier, aux aguets de toutes les fautes, surtout celles qui touchent à la sexualité, et un sentiment extrême de culpabilité. Très tôt je fus détourné de la saveur possible de Dieu, fait-elle dire à François dans le Torrent (50).

La romancière éprouve des sentiments paradoxaux, d'une part, une attirance et une admiration avouées pour la Bible, et, d'autre part, la tendance à exploiter uniquement les passages écrasants pour les humains. Le critique Gilles Marcotte voyait dans le Torrent l'image la plus forte de cette révolte contre le christianisme traditionnel au Canada français 10 .

Anne Hébert nous donne peut-être une réponse qui a une dimension à la fois idéologique et littéraire. André Vanasse, dans une interview, lui signalait le constant besoin chez elle d'accoupler le sacré et le profane (au sens de la profanation). Elle répondait que cela faisait partie de son patrimoine, parce que la liturgie, les Écritures, l'Évangile concernaient les gens de sa génération: C'étaient non seulement des dogmes, une morale, mais une culture aussi. Et à la question: Pourquoi cette insistance sur le parodique, c'est-a-dire ce besoin des formules sacrées et religieuses utilisées d̀ l'envers..., elle répondait: Peut-être que l'être humain est un être plein de contradictions... Moi je n'ai pas fait d'unité en moi. Heureusement car cela signifierait la mort de beaucoup de mes personnages 11 .

9 Faucher, Françoise, «Anne Hébert». Interview, l'Actualité, vol. 8, $\mathrm{n}^{\circ} 2$, février 1983, p. 13.

10 Marcotte, Gilles, «La religion dans la littérature canadienne-française contemporaine», in Littérature et société canadiennes-françaises, Les Presses de l'Université Laval, 1964, p. 170.

11 André Vanasse, loc. cit., p. 444. 